\title{
Self-learning Adaptive Integrated Control of an Electric Vehicle in Emergency Braking
}

\author{
Sulakshan Rajendran, Sarah Spurgeon, Georgios Tsampardoukas and Ric Hampson
}

\begin{abstract}
It is challenging to achieve high braking efficiency as well as high directional stability in emergency $\mu$-split braking manoeuvres. A self-learning adaptive integrated control scheme is presented for an electric vehicle (EV) which has a novel brake-circuit configuration. A self-learning time varying super twisting sliding mode-based anti-lock braking system (ABS) controller is integrated with a simple PID-based steering controller, adaptive super twisting sliding mode-based yaw moment controller and a yaw moment allocation module via a two-tier two-layer hierarchical scheme. The ABS controller is designed based on a model which includes the actuator dynamics, and a fuzzy module is employed to vary the slope of the sliding surface to achieve high performance levels in $\mu$-split operation. The scheme effectively executes differential braking to attain high braking performance with optimal steering effort and improved vehicle stability. Moreover, the scheme exhibits high robustness and adaptability to uncertainties and disturbances. The design has the added benefit that it is straightforward to implement in real-time. The performance of the proposed scheme is demonstrated using a $15^{\text {th }}$ - order high fidelity vehicle model whose performance has been correlated with an experimental vehicle.
\end{abstract}

\section{INTRODUCTION}

The production of EVs is ever expanding in order to respond to increasing concerns over environmental pollution and to reduce $\mathrm{CO}_{2}$ emissions. There are many challenges involved in designing the next generation of braking control systems for EVs. This is particularly true for the EV considered in this study which has only electric braking (two Individual-Wheel Motors (IWMs) on the rear driven axle and friction braking (two Electro-Hydraulic Brakes (EHB)) on the front axle. The inclusion of IWMs results in numerous additional objectives for braking control with individually driven wheels. Specifically, in addition to the design of an effective ABS controller, other objectives such as directional control must be considered to improve braking performance and to achieve a smooth driving experience. Braking in a curve or under $\mu$-split conditions is a challenging task due to the trade-off between braking efficiency and directional stability that must be achieved. Undesired lateral acceleration due to interactions between the ABS and yaw channels may

S. Rajendran and S. K. Spurgeon, University College London, Electronic and Electrical Engineering Department, London, UK.

( uceeraj@ucl.ac.uk,s.spurgeon@ucl.ac.uk).

G. Tsampardoukas and R. Hampson, Jaguar Land Rover, National Automotive Innovation Centre, Coventry, UK.

(gtsampar@jaguarlandrover.com, rhampso2@jaguarlandrover.com) cause the vehicle to understeer or oversteer and the vehicle will not follow a desired trajectory. Integrated control to enhance vehicle handling and stability has been explored [1] and it is seen that integration of different active control systems yields high performance of the vehicle. For example, Active Front Steering (AFS) and Direct Yaw Control (DYC) systems are integrated to achieve high vehicle stability and improved vehicle handling in [2] [3] [4]. However, these strategies are not intended for emergency braking manoeuvres.

Developing an efficient ABS controller is important to perform integrated control in emergency braking. It is a challenging task since the wheel slip dynamics is subject to high uncertainties and disturbances. This is because the slip dynamics depends on parameters that are highly influenced by the uncertain nonlinear tyre-road interaction dynamics. A Sliding Mode Control (SMC) based approach has been widely adopted to design an ABS controller to produce desired performance in the presence of model uncertainties, parameter variations and disturbances [5] [6] [7] [8] [9]. However, the major drawback of the reported SMC based work is that no actuator dynamics is considered in the controller design phase. Improvements in the transient performance are also required during critical $\mu$-split braking events. For industry take-up a further consideration must be the ease of real-time implementation of any scheme.

In this paper, a self-learning adaptive integrated control scheme is developed to attain optimal braking performance with high vehicle stability in critical emergency braking cases. The proposed scheme executes differential braking via the rear-driven wheels by allocating the desired moment to the rear ABS units. A self-learning time varying super twisting sliding mode-based (STW-TVSMC) ABS controller is designed based on a system model augmented by actuator dynamics to achieve high braking performance in the presence of any uncertainties and disturbance. An adaptive super twisting sliding mode-based yaw moment controller is designed to generate the desired yaw moment in the presence of any uncertainties and disturbance. Then, a control allocation module is developed to distribute the desired moment to the ABS and steering channels based on a new compound error measure which characterizes the optimal trade-off between braking efficiency and stability in emergency braking cases. 
This paper is structured in six sections. Following the introduction, the experimental vehicle and the corresponding vehicle model is presented in Section II. The cooperation principle between the braking and steering control channels is discussed in Section III. In Section IV, a self-learning adaptive integrated control scheme is presented. Then, simulation test results with the high-fidelity model are presented in Section V. Finally, conclusions are presented in Section VI.

\section{VEHICLE MODEL}

In this section, the experimental vehicle and corresponding dynamical model is presented.

\section{A. Experimental vehicle}

A unique Rear-Wheel-Driven (RWD) and Front-wheel Steering (FWS) EV with electric brakes only on the rear wheel (two electric motors (EMs)-mounted to wheel by small shaft) and friction/hydraulic brakes (EHBs) only on the front is considered in this study (see Fig.1).

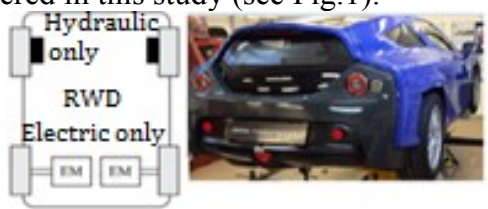

Fig.1.Experimental vehicle with novel brake-circuit configuration The torque ranges of each rear EM and the front EHB are $680 \mathrm{Nm}$ and $2000 \mathrm{Nm}$, respectively. Moreover, the rate limits of the EM and EHB are $10000 \mathrm{Nm} / \mathrm{s}$ and $2000 \mathrm{Nm} / \mathrm{s}$, respectively. The vehicle is equipped with wheel speed sensors and Inertial Measurement Units (IMU). It is powered by a $31 \mathrm{kWh} \mathrm{LiFePO}_{4}$ battery which is installed in the floor of the vehicle. A Controlled Area Network (CAN) bus is used to communicate between the Powertrain Control Module (PCM) and Sevcon Gen 4 size 8 inverters. The desired computation time of braking control system is $1 \mathrm{~ms}$.

\section{B. Vehicle model}

A $15^{\text {th }}$-order high fidelity model which has been correlated with the experimental vehicle including CAN-network model given in [10] is used for the investigation. The full car model is simplified to a 7 degree of freedom (DoF) model to design the integrated control scheme. The simplified model is described in terms of two sub systems: a 3-DoF vehicle dynamics and a 4 DoF wheel dynamics of the four wheels. The model is given by

$$
\begin{gathered}
\dot{v}_{x}=\frac{1}{m}\left(F_{x r}+F_{x f} \cos \delta-F_{y f} \sin \delta\right)+m r v_{y} \\
\dot{v}_{y}=\frac{1}{m}\left(F_{y r}+F_{x f} \sin \delta+F_{y f} \cos \delta\right)+m r v_{x} \\
\dot{r}=\frac{1}{I_{z z}}\left(l_{f} F_{x f} \sin \delta+l_{f} F_{y f} \cos \delta-l_{r} F_{y r}\right)+ \\
\frac{t_{r}}{2 I_{z z}}\left(\Delta F_{x r}+\Delta F_{x f} \cos \delta\right) \\
\dot{\omega}_{i j}=\frac{1}{J}\left(R F_{x}^{i j}-T_{b}^{i j} \operatorname{sign}\left(\omega_{i j}\right)\right)
\end{gathered}
$$

where

$$
\begin{aligned}
F_{x}^{i j} & =\mu_{x}^{i j}\left(\lambda_{i j}\right) F_{z}^{i j} \text { and } F_{y}^{i j}=\mu_{y}^{i j}\left(\alpha_{i j}\right) F_{z}^{i j} \\
\lambda_{i j} & =\frac{\left(v_{x}-\omega_{i j} R \cos \left(\alpha_{i j}\right)\right)}{v_{x}} \\
\alpha_{f} & =\tan ^{-1}\left(v_{y}+\frac{r l_{f}}{v_{y}}\right)-\delta \\
\alpha_{r} & =\tan ^{-1}\left(v_{y}-\frac{r l_{r}}{v_{y}}\right)
\end{aligned}
$$

and

$$
\begin{array}{rlrl}
F_{x f} & =F_{x}^{f L}+F_{x}^{f R} & F_{x r} & =F_{x}^{r L}+F_{x}^{r R} \\
F_{y f} & =F_{y}^{f L}+F_{y}^{f R} & F_{y r} & =F_{y}^{r L}+F_{y}^{r R} \\
\Delta F_{x r} & =F_{x}^{r L}-F_{x}^{r R} & \Delta F_{x f} & =F_{x}^{f L}-F_{x}^{f R} \\
\delta & =\delta_{f L}=\delta_{f R} & r & =\dot{\psi} \\
\alpha_{f} & =\alpha_{f L}=\alpha_{f R} & \alpha_{r} & =\alpha_{r L}=\alpha_{r R}
\end{array}
$$

where $F_{x}^{i j}$ and $F_{y}^{i j}$ are the longitudinal and lateral tyre forces, respectively of wheels ( $i j=f L$ (front left), $f R$ (front right), $r L$ (rear left), $r R$, (rear right)). $m$ is the mass of the vehicle, $\delta$ is the steering input, $r$ is the yaw rate and $v_{x}, v_{y}$ are the longitudinal and lateral velocity of the vehicle, respectively. $l_{f}$ and $l_{r}$ are respectively, the distance between front wheel to centre of gravity and real wheel to centre of gravity. The distance between the two front wheels is $t_{f}$ and the rear wheels is $t_{r} . I_{z z}$ is the moment of inertia (yaw). $\omega_{i j}$ is the angular speed of the wheels, $J$ is the wheel inertia, $R$ is the wheel radius, $\lambda_{i j}$ is the wheel slip and $\alpha_{i j}$ is the wheel side slip angle. $\mu_{x}^{i j}$ and $\mu_{y}^{i j}$ are, respectively, the longitudinal and lateral coefficients of the wheels. $T_{b}^{i j}$ is the brake torque and $F_{z}^{i j}$ is the vertical wheel load.

\section{THE PRINCIPLE OF COOPERATIVE BRAKING AND STEERING CONTROL}

The wheel slip $\left(\lambda_{i j}\right)$ of the wheel must be controlled around an optimal value $\left(\lambda_{i j}^{o p t}\right)$ to achieve high braking efficiency by utilizing the maximum friction coefficient $\left(\mu_{x, \max }^{i j}\right)$. However, the $\mu_{x, \max }^{i j}$ and corresponding $\lambda_{i j}^{o p t}$ are different for road surfaces as shown in Fig.2.

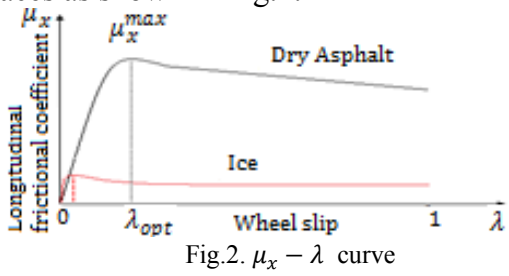

Hence, the ABS controller must be adaptive and should exhibit high robustness to different road conditions to achieve high braking performance by tracking the $\lambda_{i j}^{o p t}$ effectively in emergency braking. To study the coupling between the braking and steering channels, the $\mu_{x}-\lambda$ and $\mu_{y}-\lambda$ for a dry asphalt road is obtained for different side slip angles as shown in Fig.3a and Fig.3b, respectively. 


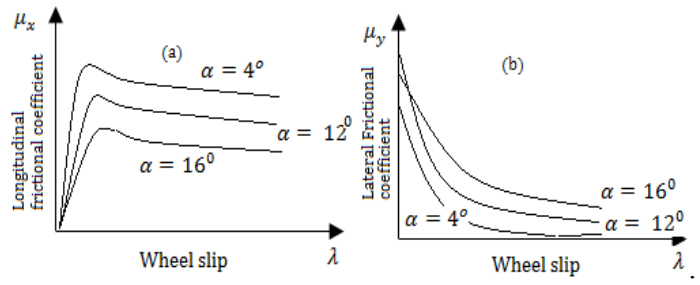

Fig.3. (a) $\mu_{x}-\lambda$ curve (b) $\mu_{y}-\lambda$ curve (dry asphalt)

It can be seen from Fig.3a that $\mu_{x}^{\max }$ reduces with increasing side slip angle. Moreover, it is evident from Fig. $3 \mathrm{~b}$ that $\mu_{y}^{\max }$ is achieved when $\lambda$ is zero and the $\mu_{y}$ decreases with increasing $\lambda$. Furthermore, $\mu_{y}^{\max }$ decreases when the side slip angle $(\alpha)$ is increased beyond an optimal value. The results are obtained for dry asphalt and similar results can be obtained for different road conditions.

It follows that during emergency braking in a curve or in a $\mu$-split event, the desired yaw moment should be generated by ensuring cooperation between the steering and ABS control channels to achieve an optimal trade-off between braking efficiency and stability. Although the differential braking of the ABS channels could produce a relatively large yaw moment when compared to the steering control, it results in low vehicle deceleration and low braking efficiency. Conversely, the steering input could produce a yaw moment without any loss of braking efficiency if operated within the linear range as seen in Fig.3. This implies that the steering input should be used as much as possible. However, if the increase in steering input produces saturation of the lateral tyre forces, the steering input will not only no longer provide more yaw moment but will also cause the vehicle to drift and result in a loss of braking efficiency. If the desired yaw moment is very large, then any further small steering input may cause a loss of steerability and stability. In this case, the ABS channels should generate the desired yaw moment, compromising on braking efficiency, to retain steerability and stability. An integrated control scheme is presented in the next section to effectively ensure cooperation between the ABS and steering controllers to achieve both high braking efficiency and directional stability.

\section{INTEGRATED CONTROL SCHEME}

The proposed scheme consists of a Steering Controller, Yaw Moment Controller, ABS controller, and a Yaw Moment Allocation Module in a two-tier two-layer hierarchy as shown in Fig.4. Since the slip dynamics is faster when compared to the vehicle dynamics, the time-varying super-twisting sliding mode- based ABS controller will be the top tier. The lower tier has two layers. The inner layer consists of a steering controller and a yaw moment controller. The outer layer employs a control allocation module to allocate the yaw moment to the ABS and steering channels. The PID-based independent steering controller generates the steering input based on the yaw rate error. An adaptive super-twisting sliding mode-based yaw moment controller is designed to generate the desired yaw moment.
A. Self-learning time varying super twisting sliding modebased ABS controller

To design the ABS controller, the slip dynamics is obtained by restricting the vehicle motion to the longitudinal axis such that $\delta=0, \alpha=0$ and $v_{y}=0$. Hence, the slip dynamics is obtained considering (1), (4), (5) and (6) as

$\dot{\lambda}_{i j}=-\frac{1}{v_{x}}\left(\left(1-\lambda_{i j}\right) \dot{v}_{x}-\frac{R^{2}}{J} F_{z}^{i j} \mu_{x}^{i j}\left(\lambda_{i j}\right)+\frac{R}{J v_{x}} T_{b}^{i j}\right)$

The actuator dynamics of the EM and EHB are modelled as $\dot{T}_{b}^{i j}=K\left(T_{b}^{i j}-\check{T}_{b}^{i j}\right)$

where $K$ is the actuator dynamic factor which characterizes the actuator properties including delay and efficiency. $\breve{T}_{b}^{i j}$ is the measured brake torque. Note that the characteristics of the actuator dynamics will vary significantly between the front and rear wheels. This actuator dynamics (11) is augmented with the slip dynamics (10) to obtain

$$
\begin{aligned}
& \dot{x}_{1}=x_{2} \\
& \dot{x}_{2}=f\left(x_{1}, x_{2}\right)+b u+d
\end{aligned}
$$

where

$$
\begin{gathered}
x=\left[x_{1} x_{2}\right]^{T}=\left[\begin{array}{ll}
\lambda_{i j} & \dot{\lambda}_{i j}
\end{array}\right]^{T} \\
f\left(x_{1}, x_{2}\right)=-\frac{1}{v_{x}}\left[-2 \dot{v}_{x} x_{2}+\ddot{v}_{x}\left(1-x_{1}\right)+\right. \\
\left.\frac{R^{2}}{J} F_{z}^{i j} \dot{\mu}_{x}^{i j}\left(x_{1}\right)+\frac{R K}{J v_{x}} \breve{T}_{b}^{i j}\right] \\
b=\frac{R K}{J v_{x}} \quad u=T_{b}^{i j}
\end{gathered}
$$

The nonlinear function $f\left(x_{1}, x_{2}\right)$ is highly uncertain. Moreover, $d$ is an external disturbance with an unknown upper bound. Hence, an adaptive super twisting-based controller is developed to track the optimal slip. The supertwisting control law (STW) is one of the most powerful second order continuous sliding mode control algorithms [11]. The major advantage of the STW is that it can alleviate chattering without compromising robustness. The adaptive STW law presented in [12] is adopted here. Considering the tracking error $e_{i j}=\lambda_{i j}-\lambda_{i j}^{o p t}$, the sliding surface is defined as

$$
\sigma_{i j}=c e_{i j}+\dot{e}_{i j}
$$

where $c$ is the slope of the surface and (13) is differentiated to obtain

$$
\dot{\sigma}_{i j}=g(x, t) u+p(x, t)
$$

where, $p(x, t)=f\left(x_{1}, x_{2}\right)+c \dot{e}_{i j}-\ddot{\lambda}_{i j}^{o p t}+d$ and $g(x, t)=b$

Assumption 1: The first-order time derivative of the uncertain function $p(x, t)$ is bounded by some unknown constant.

$$
\dot{p}(x, t) \leq P
$$

and the uncertain function $g(x, t)$ is expressed as

$$
g(x, t)=g_{0}(x, t)+\Delta g(x, t)
$$

where, $g_{0}(x, t)>0$ is the nominal part and $\Delta g(x, t)$ is the uncertain part of the function such that

$$
\left|\Delta g(x, t) / g_{0}(x, t)\right| \leq \mathfrak{g}<1
$$

With Assumption 1, (14) can be re-written as

$$
\dot{\sigma}_{i j}=p(x, t)+g_{1}(x, t) w
$$




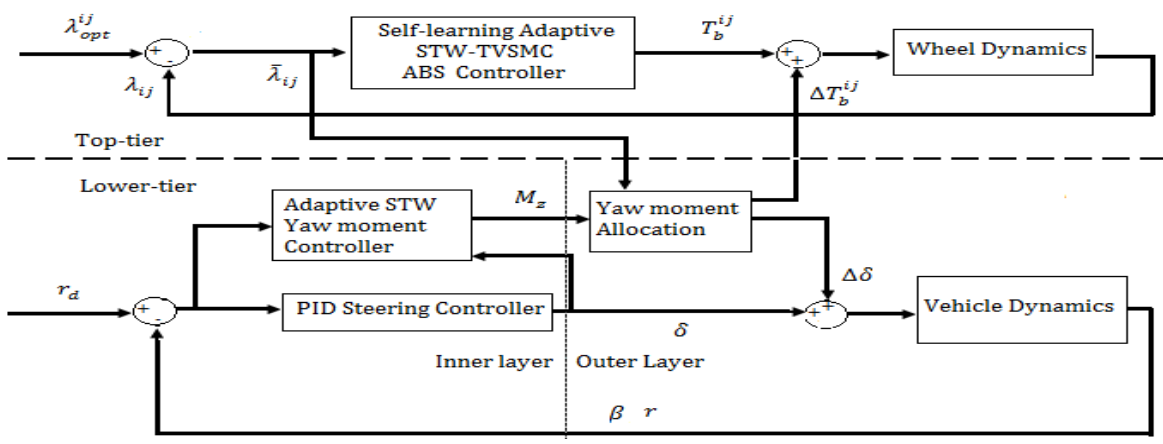

Fig.4.self-learning adaptive Integrated Control Scheme

where, $1-\mathrm{g} \leq g_{1}(x, t)=1+\Delta g(x, \iota) / g_{0}(x, t) \leq 1+\mathfrak{g}$

and $w=g_{0}(x, t) u$. The STW algorithm [11] is given as

$$
\begin{aligned}
& w=-k_{1}|\sigma|^{1 / 2} \operatorname{sign}(\sigma)+\mho \\
& \dot{\mho}=-k_{2} \operatorname{sign}(\sigma)
\end{aligned}
$$

By substituting (19) in (18) one can obtain

$$
\begin{aligned}
& \dot{\sigma}_{i j}=-k_{1} g_{1}(x, t)|\sigma|^{1 / 2} \operatorname{sign}(\sigma)+\overbrace{g_{1}(x, t) \mho+p(x, t)}^{\Upsilon} \\
& \dot{\Upsilon}=-\overbrace{2} g_{1}(x, t) \operatorname{sign}(\sigma)+\overbrace{\dot{g}_{1}(x, t) \mho+\dot{p}(x, t)}^{\Upsilon}
\end{aligned}
$$

The term $\dot{g}_{1}(x, t) \mho$ is assumed to be bounded with some unknown constant $P_{1}$; that is $\dot{g}_{1}(x, t) \mho \leq P_{1}$ and with (15)

$$
|\varpi(x, t)| \leq P+P_{1}=P_{2}
$$

The gains $k_{1}$ and $k_{2}$ are updated online by an adaptive law [12]

$$
\begin{aligned}
& k_{1}\left(\sigma_{i j}, t\right)=k_{1}^{0} \sqrt{l(t)} \\
& k_{2}\left(\sigma_{i j}, t\right)=k_{2}^{0} l(t)
\end{aligned}
$$

where $k_{1}^{0}$ and $k_{2}^{0}$ are arbitrary positive constants and the time varying positive scalar $l(t)$ is given by

$$
i(t)= \begin{cases}k & \text { if }\left|\sigma_{i j}\right| \neq 0 \\ 0 & \text { if }\left|\sigma_{i j}\right|=0\end{cases}
$$

where $k$ is a positive constant. In this way the sliding function $\sigma$ and $\dot{\sigma}$ will be driven to zero in finite time. According to (23) the gains will be increased until sliding is achieved. This avoids over estimation of the gain and high control inputs. This is important to enable precise control to maintain the slip around the optimal slip during $\mu-$ split emergency braking.

Furthermore, it is important to achieve high levels of transient performance during $\mu-$ split conditions to achieve high braking efficiency. Therefore, the slope $c$ of the sliding surface is rotated by

$$
c=\left\{\begin{array}{cl}
c_{1} & \text { if }|\sigma|>\mathcal{Q} \\
c_{1} \cdot \bar{k} & \text { if }|\sigma| \leq \mathcal{Q}
\end{array}\right.
$$

where $c_{1}$ is positive design parameter and $\bar{k}$ a factor which is adjusted by a fuzzy system to vary the desired dynamics to achieve the desired transient performance. $\mathcal{Q}$ represents a small boundary near the sliding surface. A one- dimensional, single-input single- output fuzzy logic controller is proposed to vary the factor $\bar{k}$. The input of the controller is the difference between the absolute values of the state error variables, $e_{i j}$ and $\dot{e}_{i j}: E=\left|e_{i j}\right|-\left|\dot{e}_{i j}\right|$, and its range is defined as $[-1,1]$. $\bar{k}$ is the output with range of $[0,1]$. Triangular membership functions have been selected for the input and output variables. The membership functions of the input are Negative Big (NB), Negative Medium (NM), Negative Small (NS), Zero (ZE), Positive Small (PS), Positive Medium (PM), Positive Big (PB). The membership functions of the output are Very Very Small (VVS), Very small (VS), Small (S), Medium (M), Big (B), Very big (VB), and Very Very Big (VVB). The centroid defuzzification method is used to obtain a corresponding crisp output and the rule base is given in Table 1.

Table 1. Fuzzy rule-base

\begin{tabular}{|l|l|l|l|l|l|l|l|}
\hline$E$ & NB & NM & NS & ZE & NM & VVB & NS \\
\hline $\bar{k}$ & VVB & VB & B & M & S & VS & VVS \\
\hline
\end{tabular}

\section{B. Standalone PID-based steering controller}

The lateral tyre forces are assumed to be $F_{y f}=C_{f} \alpha_{f}$ and $F_{y r}=C_{r} \alpha_{r}$ where $C_{f}$ and $C_{r}$ denote the front and rear cornering stiffness. Considering the vehicle side slip $(\beta)$

$$
\beta=\tan ^{-1} \frac{v_{y}}{v_{x}}
$$

and assuming $v_{x}$ is constant, the vehicle dynamics given by (1), (2) and (3) is simplified to a linear model

$$
\begin{aligned}
& \dot{x}=A x+B u \\
& y=r
\end{aligned}
$$

where $x=\left[\begin{array}{ll}\beta & r\end{array}\right]^{T} \quad u=[\delta]$

$$
A=\left[\begin{array}{cc}
\left(C_{f}+C_{r}\right) / m v_{x} & \left(l_{f} C_{f}-l_{r} C_{r}\right) / m v_{x}^{2} \\
\left(l_{f} C_{f}-l_{r} C_{r}\right) / I_{z z} & \left(l_{f}^{2} C_{f}+l_{r}^{2} C_{r}\right) / I_{z z} v_{x}
\end{array}\right] \quad B=\left[\begin{array}{l}
C_{f} / m v_{x} \\
l_{f} C_{f} / I_{z z}
\end{array}\right]
$$

The PID controller is designed based on the model given by (26), then tuned based on the 15-DoF nonlinear model [10]. It generates the steering input to the steering actuator based on the yaw rate error, $\tilde{r}=r-r_{d}$ where $r_{d}$ is the desired yaw rate.

\section{Yaw moment controller}

The yaw moment controller must generate the desired yaw moment $M_{z}$ which then will then be allocated to the rear ABS and steering channels, respectively. The yaw dynamics (3) is highly uncertain and nonlinear, hence, an adaptive STW 
based yaw moment controller is designed in this section. Consider the yaw dynamics given by (3) which can be rewritten as

where

$$
\dot{r}=f(\beta, r, \delta)+b u
$$

$$
\begin{gathered}
f(\beta, r, \delta)=\frac{1}{I_{z z}}\left(l_{f} F_{x f} \sin \delta+l_{f} F_{y f} \cos \delta-\right. \\
\left.l_{r} F_{y r}\right)+\frac{t_{r}}{2 I_{z z}}\left(\Delta F_{x f} \cos \delta\right) \\
b=\frac{1}{I_{z z}} \quad u=M_{z}
\end{gathered}
$$

Define the sliding surface as

$$
\sigma=r-r_{d}
$$

Equation (28) is differentiated to obtain

$$
\dot{\sigma}=g(x, t) u+p(x, t)
$$

where, $\quad p(x, t)=f(\beta, r, \delta)-\dot{r}_{d} \quad$ and $\quad g(x, t)=b$ and consider the functions $p(x, t)$ and $g(x, t)$ satisfy Assumption 1. The STW law given by (19) is substituted in (29) to obtain $\dot{\sigma}=-k_{1} g_{1}(x, t)|\sigma|^{1 / 2} \operatorname{sign}(\sigma)+\overbrace{g_{1}(x, t) \mho+p(x, t)}^{\gamma}$

$\dot{\Upsilon}=-k_{2} g_{1}(x, t) \operatorname{sign}(\sigma)+\overbrace{\dot{g}_{1}(x, t) \mho+\dot{p}(x, t)}$

Considering the condition (21) is satisfied, with the adaptive law described by (22) and (23), the sliding function $\sigma$ and $\dot{\sigma}$ will be driven to zero in finite time. Hence, the desired yaw moment $M_{z}$ is generated in the presence of uncertainties and disturbances.

\section{Yaw moment allocation module}

The desired yaw moment $M_{z}$ must be generated as a combination of the yaw moment due to the steering input $M_{z S}$ and the yaw moment generated by the differential braking of the rear-driven wheels (RWD) $M_{z d}$. Hence, the yaw moment error is defined as

$$
\widetilde{M}_{z}=M_{z}-\widehat{M}_{z}=e_{m}
$$

where $\widehat{M}_{z}=M_{z s}+M_{z d}$. As has been discussed in Section III, the yaw moment must be allocated to the ABS and steering channel to achieve high braking efficiency as well as stability. Therefore, to find an optimal trade-off, a combined error $e_{m c}$ is defined:

$$
e_{m c}^{i j}=\xi \bar{\lambda}_{i j}+e_{m}
$$

where $\bar{\lambda}_{i j}=\lambda_{i j}-\lambda_{o p t}^{i j}(i j=r R, r L)$ and $\xi$ is factor that characterizes the optimal slip range. Now the following cost function is defined

$$
J(\Gamma)=e^{T} H e+\Gamma^{T} \mathcal{F} \Gamma
$$

where $e=\left[\begin{array}{lll}e_{m} & e_{m c}^{r R} & e_{m c}^{r L}\end{array}\right]^{T} \quad \Gamma=\left[\begin{array}{lll}\bar{\delta} & \bar{T}_{b}^{r R} & \bar{T}_{b}^{r L}\end{array}\right]^{T}$

$H=\operatorname{diag}\left(\begin{array}{llll}w_{1} & w_{2} & w_{3}\end{array}\right) \quad \mathcal{F}=\operatorname{diag}\left(w_{4} \quad w_{5} \quad w_{6}\right)$ where $\Gamma$ is the vector of incremental inputs to the actuators: $\bar{\delta}=\Delta \delta, \bar{T}_{b}^{r R}=\Delta T_{b}^{r R}$ and $\bar{T}_{b}^{r L}=\Delta T_{b}^{r L} . w_{1}, w_{2} w_{3}$ are the weighting factors of error vector $e$ and $w_{1} \quad w_{2} \quad w_{3}$ are the weights of the control inputs. The weights are selected considering the coupled nature of the steering and braking channels discussed in Section III. The weights are optimized based on the optimal slip range. Considering the practical implementation, the following constraints are defined.

$$
\mu_{i j}^{2} \geq F_{x}^{i j^{2}} /_{F_{z}^{i j^{2}}}+F_{y}^{i j^{2}} /_{F_{z}^{i j^{2}}}, T_{b \min }<T_{b}<T_{b \max }
$$

$\dot{T}_{\text {bmin }} \leq \dot{T}_{b} \leq \dot{T}_{\text {bmax }}, \dot{\delta}_{\text {bmin }} \leq \dot{\delta}_{b} \leq \dot{\delta}_{\text {bmax }}$

where $\dot{T}_{b}$ and $\dot{\delta}_{b}$ are the actuation rates of braking actuators and steering actuator and $\mu_{i j}$ is the friction limit per wheel. The inputs of the last step are used to calculate the constraints, $\Omega_{k}(\Gamma)(k=1 \ldots \ldots n)$. Hence, the optimization problem (33) can be re-written as

$$
\begin{array}{ll} 
& \min J(\Gamma) \\
\text { s.t. } & \Omega_{k}(\Gamma) \geq 0(k=1 \ldots . n)
\end{array}
$$

The augmented Lagrangian method [13] has been chosen for optimization due to its convergence properties. Therefore, the optimization problem (35) is described by the augmented Lagrangian function

$$
\begin{aligned}
\min \Phi\left(\Gamma, w, \eta_{p}\right)= & J(\Gamma)+1 / 2 \eta_{p} \sum_{k=1}^{n}\left\{\left[\operatorname { m a x } \left(0, w_{k}-\right.\right.\right. \\
& \left.\left.\eta_{p} \Omega_{k}(\Gamma)\right]^{2}-w_{k}^{2}\right\}
\end{aligned}
$$

where $\eta_{p}$ is the penalty factor. Finally, the desired change in brake torque $\Delta T_{b}^{i j}$ and steering input $\Delta \delta$ is added to the brake torque $T_{b}^{i j}$ generated by ABS controller and the PID steering controller, respectively, as shown in the Fig.4.

\section{SIMULATION RESULTS}

The performance of the designed self-learning adaptive integrated control scheme has been evaluated via simulation tests with a 15-order high fidelity full car nonlinear model [10]. In the simulation tests, the unmeasurable states (optimal slip, slip, vehicle velocity, tyre forces, desired yaw rate) are estimated using the adaptive higher order sliding mode observer presented in [10]. A critical emergency $\mu-$ split braking case in a curved path is simulated to assess the performance of the proposed scheme.

The vehicle starts braking from $30 \mathrm{~m} / \mathrm{s}$ on a surface of $\mu=1$ and then after 2 seconds of braking, the road track exhibits $\mu-$ split behavior at the turn so that on the the right track $\mu=0.2$ and on the left track $\mu=1$. Moreover, noises are added to the sensor signals. The desired trajectory of the vehicle is illustrated in Fig.5a. The desired stopping time is 8 seconds. The obtained results are shown in Fig.6. It can be seen from Fig.6a that the vehicle has stopped in 8 seconds effectively tracking the desired trajectory (Fig.5b). The timevarying super twisting sliding mode based-ABS controller has efficiently tracked the desired wheel slips (Fig.6b and Fig .6c) exhibiting high robustness to actuator (EM and EHB) and road uncertainties. It can be seen from Fig.6d that the differential braking has been executed via the rear braking channels to maintain the desired trajectory with smooth steering effort (Fig.6f). The standalone steering controller (without integration with the ABS) has failed to track the desired trajectory and yaw rate as seen from Fig.5a and Fig.6e, respectively. Furthermore, the integrated control scheme exhibits smooth and reduced steering effort when compared to the standalone steering controller (Fig.6f). 


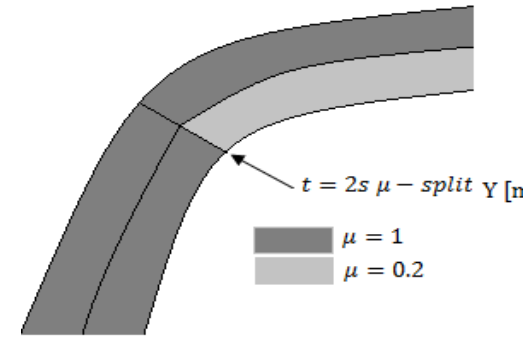

(a)

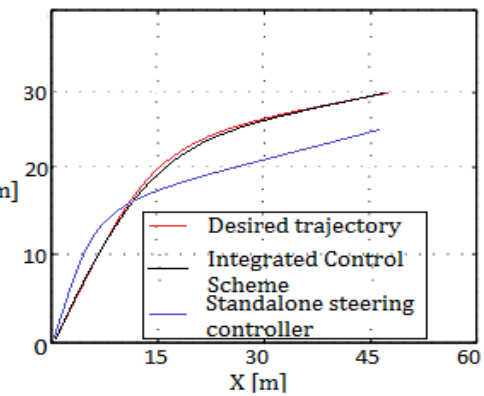

(b)

Fig.5 (a) Illustration of braking path

(b) vehicle trajectory

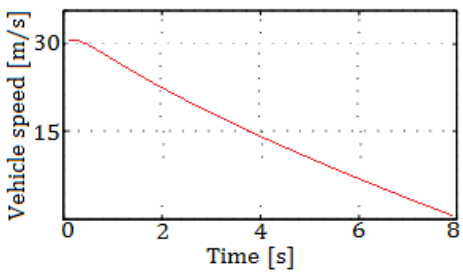

(a)

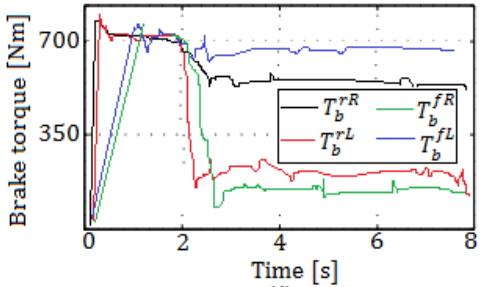

Time

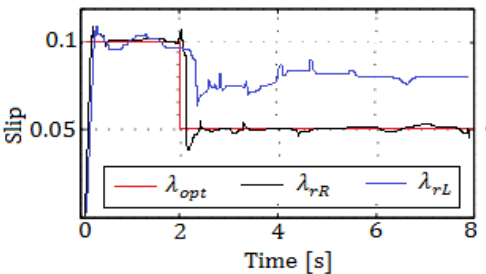

(b)

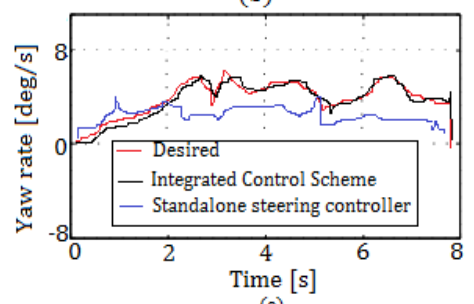

(e)
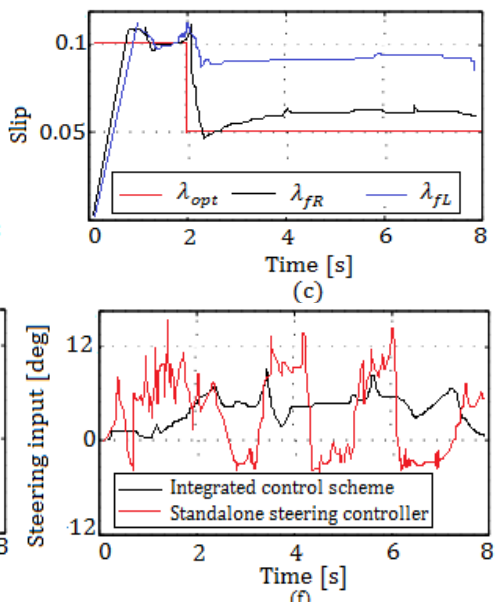

Time $[s]$

Fig.6 (a) vehicle speed (b) rear wheel slips (c) front wheel slips (d) brake torques (e) yaw rate (f) steering input

\section{CONCLUSIONS}

The proposed self-learning adaptive integrated control scheme has achieved high performance during emergency braking in a curved trajectory in the presence of uncertainties and disturbances. Moreover, the proposed self-learning adaptive STW-TVSMC ABS controller has exhibited exceptionally good transient performance during a critical $\mu$ split event. The significance of the proposed scheme is that it can easily be adapted to different actuators with varying actuator characteristics. Moreover, the design is straight forward, easy to tune and can easily be implemented in realtime. In future, a fuzzy based yaw moment allocation module will be investigated and will be compared with the augmented Lagrangian approach with respect to computational effort.

\section{REFERENCES}

[1] T. Gordon, M. Howell, and F. Brandao, "Integrated control methodologies for road vehicles," Vehicle System Dynamics, Vol. 40 No. 1-3, pp. 157-190, 2003.

[2] Y. Chen, J. Hedrick, and K. Guo, "A novel direct yaw moment controller for in-wheel motor electric vehicles," Vehicle System Dynamics, vol. 51, no. 6, pp.925-942, 2013.

[3] J. Wu, S. Cheng and B. Liu, "A human-machine-cooperative-driving controller based on AFS and DYC for vehicle dynamic stability". Energies, Vol. 10, Issue 11, p. 1737,2017.
[4] A. T. Johansen, "Integration of vehicle yaw stabilization and rollover prevention through nonlinear hierarchical control allocation. Vehicle System Dynamics, Vol. 52, Issue 12, p. 1607-1621, 2014.

[5] M. Amodeo, A. Ferrara and R. Terzaghi, "Wheel slip control via second-order sliding-mode generation. IEEE Transactions on Intelligent Transportation Systems :11(1): 122-131, 2010.

[6] G. P. Incremona, E. Regolin, A. Mosca and A. Ferrara, "Sliding mode control algorithms for wheel slip control of road vehicles", Proc. Am. Control Conf., pp. 4297-4302, 2017.

[7] M. Savaresi and M. Tanelli, Active Braking Control Systems Design for Vehicles, Berlin, Germany: Springer, 2010.

[8] J. Zhang and J. Li, "Adaptive backstepping sliding mode control for wheel slip tracking of vehicle with uncertainty observer", Meas. Control, vol. 51, no. 9-10, pp. 396-405, 2018.

[9] R. Verma, D. Ginoya, P. Shendge and S. Phadke, "Slip regulation for anti-lock braking systems using multiple surface sliding controller combined with inertial delay control", Vehicle System Dynamics, vol. 53, no. 8, pp. 1150-1171, 2015.

[10] S. Rajendran, S. K. Spurgeon, G. Tsampardoukas and R. Hampson "Estimation of road frictional force and wheel slip for effective antilock braking system (ABS) control". International Journal of Robust and Nonlinear Control .29(3), c. 736-765, 2019.

[11] A. Levant, "Higher-order sliding modes, differentiation and output-feedback control," International Journal of Control, vol. 76, no. 9-10, pp. 924-941, 2003.

[12] Y. Zheng, J. Liu, X. Liu, D. Fang, L. Wu, "Adaptive second-order sliding mode control design for a class of nonlinear systems with unknown input. Math. Probl. Eng. p,7, 2015.

[13] R. Andreani, E. G. Birgin, J. M. Martínez, and M. L. Schuverdt, "On Augmented Lagrangian methods with general lower-level constraints," SIAM J. Optim., Vol. 18, No. 4, pp. 1286-1309, 2007. 